\title{
Qualidade de vida e adiposidade em adolescentes: efeitos diretos e indiretos
}

\author{
Quality of life and adiposity in adolescents: \\ direct and indirect effects
}

Isabelle Arruda Barbosa (http://orcid.org/0000-0003-3121-0505) ${ }^{1}$

Marise Fagundes Silveira (https://orcid.org/0000-0002-8821-3160) ${ }^{2}$

Adélia Dayane Guimarães Fonseca (https://orcid.org/0000-0002-1168-7106) ${ }^{3}$

Maria Fernanda Santos Figueiredo Brito (https://orcid.org/0000-0001-5395-9491) ${ }^{2}$

Lucinéia de Pinho (https://orcid.org/0000-0002-2947-5806) ${ }^{2}$

Carla Silvana de Oliveira e Silva (https://orcid.org/0000-0002-2752-1557) ${ }^{2}$

${ }^{1}$ Instituto Federal do Norte de Minas Gerais. Rodovia BR 367, km 07, s/n, Zona Rural. 39900-000 Almenara MG Brasil. isabelleab3@gmail.com ${ }^{2}$ Universidade Estadual de Montes Claros. Montes Claros MG Brasil.

${ }^{3}$ Universidade Federal de Juiz de Fora. Juiz de Fora MG Brasil.

\begin{abstract}
This paper aimed to analyze the direct and indirect effects of adiposity and factors related to adolescents' quality of life. This is a cross-sectional study with 635 adolescents (10-16 years) from public schools in Montes Claros-MG, Brazil. Anthropometric data, physical activity (PA), inadequate dietary habits, body image, and quality of life (QoL) of adolescents were collected. A structural equation modeling was performed. The variables addressed as constructs were adiposity and inadequate dietary habits, the exploratory variables were PA and body image, and the outcome variable was QoL. The total effect of PA-mediated adiposity on QoL was positive and significant $(\beta=0.213 ; p<0.05)$, in contrast to the total effect mediated by inadequate dietary habits on QoL, which was negative and significant ( $\beta=-0.150$; $p<0.05)$. The direct effect of PA on QoL was positive and significant $(\beta=0.209 ; p<0.001)$. No body image-related effects were observed. The effects of adiposity on adolescents' QoL tend to improve when mediated by $P A$, and they worsen when mediated by inadequate dietary habits. The effects of $P A$ tend to improve the QoL of adolescents.
\end{abstract}

Key words Adolescents, Multivariate analysis, Quality of life, Adiposity, Risk factors
Resumo Objetivou-se analisar os efeitos diretos $e$ indiretos da adiposidade e de fatores relacionados à qualidade de vida de adolescentes. Estudo transversal realizado com 635 adolescentes (10 a 16 anos), de escolas públicas da cidade de Montes Claros-MG. Coletaram-se dados antropométricos, de atividade física $(A F)$, hábitos alimentares inadequados, imagem corporal e qualidade de vida $(\mathrm{QV})$ dos adolescentes. Foi realizada a modelagem com equações estruturais. As variáveis tratadas como construto foram adiposidade e hábitos alimentares inadequados, as variáveis exploratórias foram $A F$ e imagem corporal; a variável desfecho, QV. O efeito total da adiposidade mediada pela $A F$ sobre a $Q V$ foi positivo e significativo $(\beta=0,213 ; p<0,05)$, em contraste com o efeito total mediado pelos hábitos alimentares inadequados sobre a $Q V$, que foi negativo e significativo $(\beta=-$ $0,150 ; p<0,05)$. O efeito direto da $A F$ sobre a $Q V$ foi positivo e significativo $(\beta=0,209 ; p<0,001)$. Não se observou efeitos relacionados à imagem corporal. Os efeitos da adiposidade tendem a melhorar a $\mathrm{QV}$ dos adolescentes, quando mediados pela prática de AF e a piorá-la, quando mediados pelos hábitos alimentares inadequados; os efeitos da AF tendem a melhorar a $Q V$ dos adolescentes. Palavras-chave Adolescentes, Análise multivariada, Qualidade de vida, Adiposidade, Fatores de risco 


\section{Introdução}

Com a redução das doenças infecciosas, o excesso de peso corporal tem configurado em um dos principais problemas de saúde pública mundial, com aumento de sua prevalência nos últimos anos $^{1}$. Todavia, a preocupação para com esse evento em idades mais jovens merece ênfase, uma vez que a obesidade obtida durante a infância e adolescência tende a continuar na idade adulta ${ }^{2}$.

Estudos advertem que tanto na adolescência quanto na fase adulta, o indivíduo adquire vários comportamentos ou estilo de vida ditos não saudáveis, de modo simultâneo, destacando-se a inatividade física e o consumo inadequado de frutas, verduras e legumes ${ }^{3,4}$.

Conquanto grande parte do interesse científico e de saúde se concentre em avaliar as implicações fisiológicas do excesso de peso na saúde, a deterioração crônica nas capacidades funcionais, sociais e psicológicas também tem sido percebi$\mathrm{da}$, impactando na qualidade de vida (QV) das pessoas $^{5-7}$. Crianças e adolescentes obesas exibem pior QV e maior insatisfação com a imagem corporal, ao serem comparadas a crianças e adolescentes de peso saudável ${ }^{8}$. Os transtornos psicossociais relacionados à obesidade compreendem ainda ansiedade, depressão, angústia, baixa autoestima, sentimento de culpa, dificuldades de ajustamento social e desordens comportamentais. Tais condições podem culminar em causa ou efeito do processo de ganho de peso, com possíveis repercussões negativas na $\mathrm{QV}^{9,10}$.

É notório o número de pesquisas que buscam estudar as associações entre a QV e excesso de peso $^{6-8,11}$, todavia pretende-se discutir no presente estudo, as relações que compõem o fenômeno de modo direto e indireto e por meio dos fatores de riscos clínicos, estilo de vida e imagem corporal, algo ainda não descrito na literatura. A compreensão dessas relações que impactam direta ou indiretamente na QV poderá tornar mais abrangente o estudo acerca dessa relevante questão de saúde pública.

Diante disso, as hipóteses testadas neste estudo foram de que a adiposidade produz efeito direto e negativo sobre a QV de adolescentes e produz efeitos indiretos e negativos, mediados pelo estilo de vida e imagem corporal, sobre a QV de adolescentes. Assim, o objetivo deste estudo foi analisar os efeitos diretos e indiretos da adiposidade e de fatores de risco sobre a qualidade de vida de adolescentes.

\section{Métodos}

Trata-se de um estudo transversal realizado com adolescentes com idade de 10 a 16 anos, do ensino fundamental $\left(5^{\circ}\right.$ ao $9^{\circ}$ ano) e médio ( $1^{\circ} \mathrm{e}$ $2^{\circ}$ ano), oriundos de escolas públicas urbanas da cidade de Montes Claros, Minas Gerais, Brasil, em 2016.

O tamanho da amostra foi definido com a finalidade de estimar parâmetros populacionais com prevalência de 0,50 , para assegurar maior tamanho amostral. Os níveis de confiança e margem de erro adotados foram de $95 \%$ e $5 \%$, respectivamente. A correção para população finita, bem como para a correção para o efeito do desenho foram realizadas, assumindo-se deff igual a 1,5. Um acréscimo de $10 \%$ foi instituído objetivando-se compensar as possíveis não-respostas e perdas ${ }^{12}$. Os cálculos evidenciaram a necessidade de se examinar e entrevistar, no mínimo, 634 adolescentes.

Os adolescentes foram selecionados por amostragem probabilística por conglomerado em dois estágios. No primeiro estágio, foram selecionadas por amostragem proporcional ao tamanho, cinco escolas representantes das quatro regiões da cidade (norte, sul, leste e oeste). Os critérios de elegibilidade das escolas foram: (i) públicas estaduais, (ii) nível escolar fundamental e médio, (iii) não participantes de qualquer pesquisa científica envolvendo prática regular de atividade física (AF), no momento da pesquisa. No segundo estágio, sortearam-se os adolescentes por amostragem aleatória sistematizada, em todas as turmas de cada escola selecionada, com base na lista de frequência.

Foram excluídos do estudo, dezenove adolescentes com relatos de doença inflamatória e infecciosa importantes, em uso de drogas que afetam o perfil metabólico e hemodinâmico, adolescentes grávidas, e aqueles que não compareceram no dia da coleta. Esses adolescentes foram substituídos por outros que, estando presentes, os antecediam ou precediam na lista de frequência escolar e que aceitaram participar da pesquisa. Conforme os critérios supracitados, a amostra final, respeitando a proporcionalidade para sexo e idade, foi composta por 635 adolescentes com representatividade populacional (de um total de 77.833 estudantes).

Diretores das escolas participantes e os pais e/ou responsáveis permitiram a participação dos adolescentes no estudo por meio de um consentimento informado. Do mesmo modo, os próprios adolescentes assentiram sua participação 
por meio de um termo de assentimento, após receberem informações relacionadas.

A coleta de dados aconteceu nas respectivas escolas dos adolescentes, no segundo semestre de 2016, feita por uma equipe multidisciplinar. Esta equipe foi composta por profissionais médicos, enfermeiros, nutricionistas, profissional de educação física, fisioterapeuta, auxiliados por estudantes da graduação na área da saúde, após treinamento e calibração. Os profissionais tidos como aptos para a coleta de dados demonstraram ter desenvolvido uma padronização nos critérios de aferição de dados antropométricos investigados (Kappa $>0,60$ ), além do acaso.

A aferição do peso dos adolescentes foi realizada em balança portátil com escala digital, capacidade de $150 \mathrm{~kg}$, precisão de $0,1 \mathrm{~kg}$ (Modelo Body complete, com 8 eletrodos e interface modelo. BF 100 - Beurer, Ulm, Alemanha). Para medir a altura, foi usado um estadiômetro portátil com haste de medição dobrável e tripé de apoio, capacidade de medição de $115 \mathrm{~cm}$ a $210 \mathrm{~cm}$, tolerância: $+/-2$ $\mathrm{mm}$ em $210 \mathrm{~cm}$, e resolução em milímetros (Modelo professional Sanny ${ }^{\circledR}$, São Paulo, Brasil). Os dados de altura $(\mathrm{cm})$ e peso $(\mathrm{kg})$ foram utilizadas para calcular o índice de massa corporal (IMC) (peso $(\mathrm{kg}) /$ altura $\left.\left(\mathrm{m}^{2}\right)\right)^{13}$. A circunferência da cintura (CC) foi avaliada com uma trena antropométrica, inelástica e flexível, sem trava, com limite de $2 \mathrm{~m}$ e precisão de $0,1 \mathrm{~cm}$, foi medida na porção mais estreita do tronco, entre a margem costal inferior e a crista ilíaca ${ }^{13}$. A razão cintura estatura (RCEst) foi calculada utilizando-se dados de CC e altura $(\mathrm{CC}(\mathrm{cm}) / \text { altura }(\mathrm{cm}))^{13}$.

Aplicaram-se os seguintes questionários para coletar informações dos adolescentes: questionário para coleta de informações demográficas; o KIDSCREEN-27 $7^{14}$, para avaliação da QV dos adolescentes; o Questionário Internacional de Atividade Física (IPAQ) - versão $\operatorname{curta}^{15}$, para identificar a duração e a frequência de AF; o Teste: como está a sua alimentação? ${ }^{16}$, do Ministério da Saúde, para informações quanto aos hábitos alimentares inadequados; e a Escala de Silhue$\operatorname{tas}^{17}$, para identificar a autopercepção da imagem corporal. Ressalta-se que os adolescentes, foram devidamente orientados a respeito da maneira correta do preenchimento dos questionários e estimou-se um tempo médio de 20 minutos para seu preenchimento.

A variável QV (numérica contínua) além de constituir-se no desfecho deste estudo, foi utilizada como variável observada, sendo investigada através do KIDSCREEN-27 $7^{14}$. Este instrumento avalia a QV relacionada à saúde de crianças $\mathrm{e}$ adolescentes, sendo composto por cinco dimensões: Bem-estar físico (5 itens), Bem-estar psicológico ( 7 itens), Autonomia e relação com os pais (7 itens), Pares e suporte social (4 itens) e Ambiente escolar (4 itens). Todos os itens estavam em escala tipo Likert e buscavam identificar a frequência de comportamentos/sentimentos ou, em determinados casos, a intensidade de atitudes específicas, com período recordativo da semana que antecede a aplicação do questionário. Os escores de cada dimensão (que variam de 1 a 5) foram computados por meio de média aritmética simples e o escore final equivalente a cada dimensão. Quanto maior o valor, maior a percepção do indicador de QV da dimensão em questão ${ }^{14}$, com exceção do item 1 da dimensão Bem-estar físico e dos itens 4,5 e 6 da dimensão Bem-estar psicológico que originalmente apresentam escores invertidos.

A variável AF (variável numérica contínua), tratada como variável observada, foi investigada através do IPAQ - versão curta ${ }^{15}$. Este questionário é composto por sete questões abertas e suas informações permitem estimar a duração e frequência por semana, gasta em diferentes intensidades de AF (domésticas, de intensidades moderada e vigorosa) e de inatividade física (posição sentada). $\mathrm{O}$ escore final foi apresentado em equivalentes metabólicos (MET) em minutos por semana, sendo calculado pelo produto da frequência pela duração de $\mathrm{AF}$, para cada intensidade de $\mathrm{AF}$, seguido da soma do resultado de todas as intensidades de AF. Quanto maior a duração e a frequência da $\mathrm{AF}$ por semana, melhor o nível de AF (caminhada $=3$ $\mathrm{METs} / \mathrm{min}$; atividades moderadas $=4 \mathrm{METs} / \mathrm{min}$; atividades vigorosas $=8 \mathrm{METs} / \mathrm{min})^{18}$.

A imagem corporal (variável numérica discreta), foi tratada como variável observada e investigada por meio da escala de silhuetas ${ }^{17}$, com perfis que vão desde a magreza (silhueta 1) até a obesidade severa (silhueta 9). Nessa escala, o indivíduo elegeu o número da silhueta que julgou ser semelhante a sua aparência corporal real, bem como com sua aparência corporal ideal. O escore final foi calculado pela diferença entre a aparência real e a ideal, sendo apresentado em valores absolutos, que variam de -8 a 8 , sendo indivíduo classificado como satisfeito se igual a zero e insatisfeito, se diferente de zero. Diante da diferença positiva, considerou-se insatisfação pelo excesso de peso e, quando negativa, insatisfação pela magreza.

As variáveis IMC, CC, RCEst também foram tratadas como variáveis observadas. Para classificar os adolescentes quanto ao IMC, utilizaram- 
se parâmetros da OMS (escore $\mathrm{Z} \geq 1$ ) para idade e sexo ${ }^{19}$. Para classificação dos valores de CC e percentual de gordura na região central, considerou-se o percentil $75 \mathrm{da}$ amostra, conforme sexo e idade ${ }^{18}$. Procedeu-se ao cálculo da RCEst pela razão da medida do perímetro da cintura $(\mathrm{cm}) \mathrm{e}$ a estatura $(\mathrm{cm})$, considerando-se como risco aumentado, valores $>0,5^{19}$.

As seguintes variáveis foram tratadas como construto, variáveis mensuradas por meio da variância compartilhada de outras variáveis, diretamente observadas: (a) adiposidade, mensurada por três variáveis numéricas contínuas: IMC, CC e RCEst; (b) hábitos alimentares inadequados, mensurada usando-se duas questões do instrumento Teste: como está a sua alimentação? ${ }^{\text {?6 }}$, do Ministério da Saúde. Este teste é composto por 18 questões, com opções de resposta variando entre 2 e 5 alternativas, referentes ao consumo habitual em porções, de itens dos grupos da pirâmide alimentar: óleos, verduras, frutas e legumes, proteínas carboidratos e água; como também do consumo de álcool, sal; e prática de atividade física ${ }^{16}$. As questões 10 e 11 deste teste foram utilizadas neste estudo e intituladas de "ultraprocessados e "guloseimas", respectivamente. São elas: (10) "Pense nos seguintes alimentos: frituras, salgadinhos fritos ou em pacotes, carnes salgadas, hambúrgueres, presuntos e embutidos (salsicha, mortadela, salame, linguiça e outros). Você costuma comer qualquer um deles com que frequência?" ${ }^{16}$, neste estudo denominada de "ultraprocessados"; e (11) "Pense nos seguintes alimentos: doces de qualquer tipo, bolos recheados com cobertura, e biscoitos doces, refrigerantes e sucos industrializados. Você costuma comer qualquer um deles com que frequência?"16, neste estudo denominada de "Guloseimas". Ambas as questões apresentam-se em escala tipo Likert (Todos os dias $=0$; De 4 a 5 vezes por semana $=1$; De 2 a 3 vezes por semana $=2$; Menos que 2 vezes por semana $=3$; Raramente ou nunca $=4$ ), sendo que quanto menor o escore da opção de resposta, maior a frequência de hábitos alimentares inadequados referida pelo adolescente.

Foi elaborado um modelo teórico visando identificar as inter-relações entre a variável desfecho QV e as demais variáveis envolvidas neste estudo. Nele, as variáveis observadas estão representadas por retângulos, os construtos, por elipses, e as associações, por setas ou trajetórias (partindo-se da variável independente para a dependente $)^{20}$ (Figura 1).

As variáveis observadas ordinais foram descritas por meio de suas distribuições de frequên- cias (absoluta e relativa) e as variáveis observadas numéricas, pelas medidas de tendência central e dispersão (média e desvio padrão), com correção pelo efeito do desenho (deff). A normalidade das variáveis foi analisada pelos coeficientes de assimetria (Sk) e curtose (Ku), cujos valores absolutos superiores a três e sete, respectivamente, indicaram desvio severo deste pressuposto ${ }^{20}$. A variável AF apresentou desvios severos da normalidade e foi submetida à transformação logarítmica, além de apresentar dados faltantes superiores a $10 \%$, que por esta razão, foram imputados pela regressão linear.

$\mathrm{O}$ ajuste do modelo ocorreu em duas etapas. Primeiramente ajustou-se os modelos de mensuração dos construtos adiposidade e hábitos alimentares inadequados através da análise fatorial confirmatória, no qual o peso fatorial acima de 0,4 foi considerado adequado ${ }^{20}$. Procedeu-se então ao ajuste do modelo multivariado, a partir da modelagem com equações estruturais (MEE) (Figura 2). Efeitos diretos foram estimados através de coeficientes padronizados $(\mathrm{CP})$, cuja significância foi analisada pela relação entre o valor do coeficiente e seu erro-padrão (razão crítica - RC), ao nível de $5 \%$. Os efeitos indiretos, mediados por variáveis intermediárias, foram calculados multiplicando-se os coeficientes de todas as rotas indiretas do modelo. Foram calculadas ainda as porcentagens das variâncias (explicadas e manifestas dos construtos). Os CP foram interpretados segundo recomendações de Kline ${ }^{21}$, onde valores próximos a 0,10 indicaram efeito pequeno, valores próximos de 0,30 indicaram efeito médio e valores maiores de 0,50 , efeito grande.

Para verificação da qualidade do modelo, foram empregados índices que verificam a intensidade ou o grau que o modelo prediz a matriz de covariância, permitindo a comparação do modelo proposto ao modelo nulo. Esses índices de verificação podem ser subdivididos em índices de ajuste geral: qui-quadrado sobre os graus de liberdade $\left(\chi^{2} / \mathrm{df}\right)$ e; e índices comparativos: de Bentler (CFI), de bondade do ajuste (GFI), de Tucker-Lewis (TLI) e erro quadrático médio (RMSEA). O ajuste do modelo aos dados foi considerado adequado quando foram obtidos pesos de itens fatoriais $\lambda \geq 0,50 ; \chi^{2} / \mathrm{df} \leq 4,0$; GFI, CFI e TLI $\geq 0,90$ e RMSEA $\leq 0,10^{20}$.

As análises descritivas das variáveis foram obtidas através do Software Package for the Social Sciences (IBM SPSS 22.0) e a construção gráfica do modelo, por meio do programa Analysis of Moment Structures (IBM SPSS AMOS 22.0), criado especificamente para realizar MEE. 


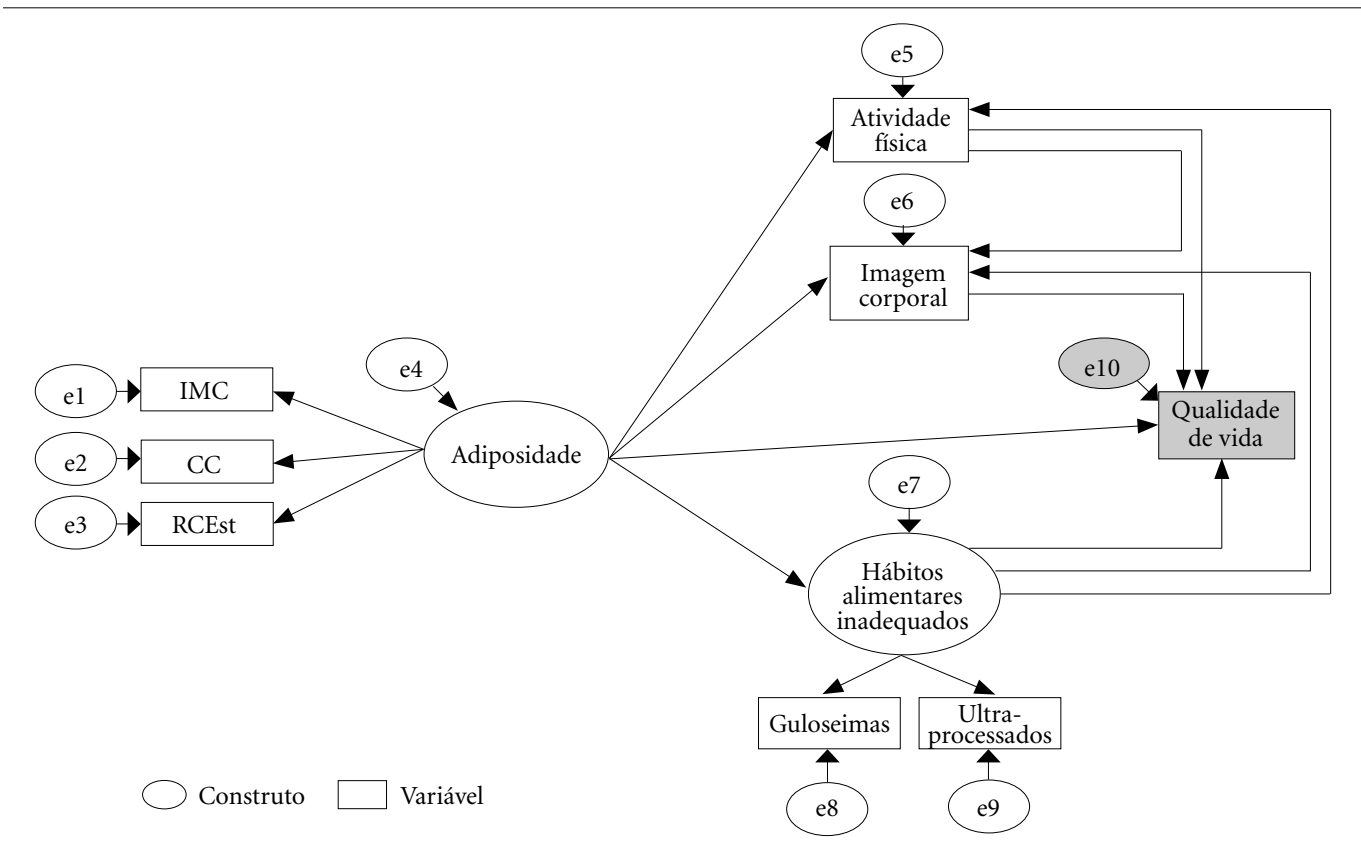

Figura 1. Modelo teórico testado para avaliar os efeitos diretos e indiretos da adiposidade e de fatores relacionados sobre a qualidade de vida de adolescentes de 10 a 16 anos. Montes Claros, Minas Gerais, Brasil, 2016.

IMC - índice de massa corporal (escore Z); CC - circunferência da cintura (cm); RCEst - Razão cintura estatura (cm).

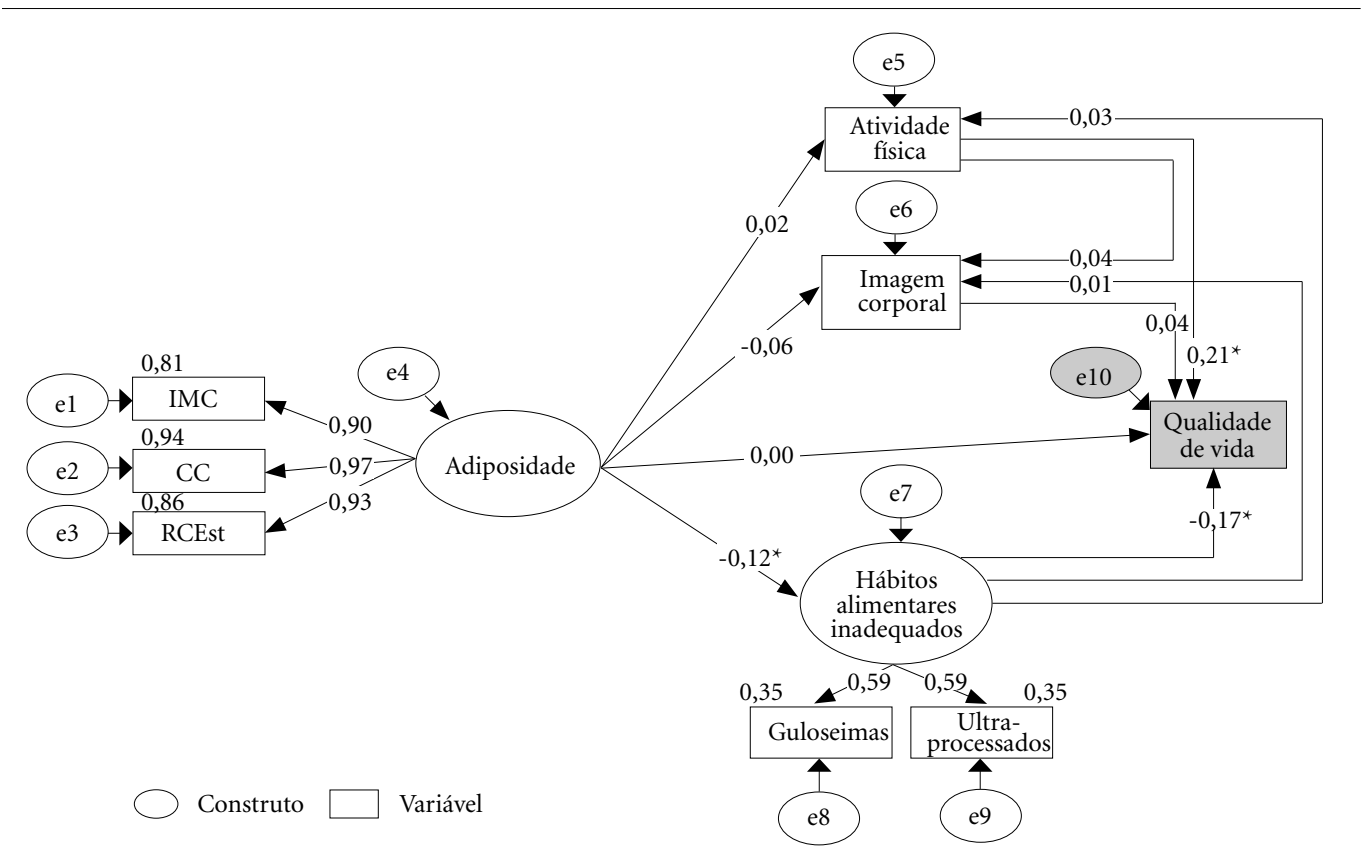

Figura 2. Modelo com equações estruturais ajustado para avaliar os efeitos diretos e indiretos da adiposidade e de fatores relacionados sobre a qualidade de vida de adolescentes de 10 a 16 anos. Montes Claros, Minas Gerais, Brasil, 2016.

IMC - índice de massa corporal (escore Z); CC - circunferência da cintura (cm); RCEst - Razão cintura estatura (cm). ${ }^{*}$ p-valor $<0,05$. 
Este estudo adotou os critérios da Resolução no 466/2012 do Conselho Nacional de Saúde e as suas resoluções complementares. O projeto foi aprovado pelo Comitê de Ética em Pesquisa da Universidade Estadual de Montes Claros.

\section{Resultados}

Participaram do estudo 635 adolescentes de escolas públicas da área urbana. A média da idade foi de $13,8( \pm 1,7)$ anos, com maioria do sexo feminino $(60,2 \% ; n=382)$.

Quanto aos hábitos alimentares inadequados, 32\% $(n=203)$ dos adolescentes responderam que consomem "ultraprocessados" de duas a três vezes/semana e $29,1 \%(n=185)$ consomem "guloseimas" menos que duas vezes/semana (Tabela 1). Na Tabela 1 apresentam-se os dados descritivos das demais variáveis do estudo.

A Figura 2 apresenta o modelo estrutural ajustado, que revelou uma boa qualidade de ajuste: $\chi^{2} / \mathrm{df}=1,901 ; \mathrm{CFI}=0,995 ; \mathrm{GFI}=0,990 ; \mathrm{TLI}=0,989$; RMSEA=0,038 (IC90\%: 0,013-0,060).

As trajetórias dos construtos que apresentaram CF mais elevadas $(>0,50)$ e significativas, foram: $C C \leftarrow$ adiposidade $(\beta=0,97 ; \mathrm{p}<0,001)$ com 94\% da variabilidade da CC dos adolescentes avaliados, explicada por este construto; e ambas trajetórias: guloseimas $\leftarrow$ hábitos alimentares inadequados e industrializados $\leftarrow$ hábitos alimentares $(\beta=0,59 ; \mathrm{p}<0,005)$, cada qual com $35 \%$ da variabilidade da variável hábitos alimentares inadequados investigada nos adolescentes, explicada por este construto (Figura 2, Tabela 2).

Quanto à magnitude dos efeitos verificou-se que o efeito total (direto + indireto) da adiposidade na $\mathrm{QV}$, mediada pela $\mathrm{AF}$ foi pequeno, positivo e significativo; o efeito total da adiposidade na QV, mediada por hábitos alimentares inadequados foi pequeno, negativo e significativo, porém foi menor que o efeito direto. $\mathrm{O}$ efeito direto da AF na QV foi pequeno, positivo e significativo; e o efeito direto de hábitos alimentares inadequados na QV foi pequeno, negativo e significativo. Os demais efeitos não se mostraram significativos (Tabela 3).

\section{Discussão}

Tendo-se em vista a complexidade existente em torno do excesso de peso corporal (EPC), este estudo objetivou analisar os efeitos diretos e indiretos da adiposidade e de fatores de risco sobre a qualidade de vida de adolescentes.

A análise revelou que dentre as variáveis do modelo, a AF exerceu efeito pequeno, positivo e significativo sobre a QV dos adolescentes. Diversos estudos que investigaram a $\mathrm{QV}$ de adolescentes relacionada à prática de $\mathrm{AF}$, corroboraram com estes resultados e concluíram que um maior nível de AF está associado a maiores escores de $\mathrm{QV}^{22,23}$. Estudo que investigou dentre outros, as associações do nível de AF e IMC com o domínio físico da QV de adolescentes, verificou que indi-

Tabela 1. Análise descritiva das variáveis do modelo com equações estruturais utilizando a qualidade de vida como variável desfecho, entre adolescentes de 10 a 16 anos. Montes Claros, Minas Gerais, Brasil, 2016 ( $\mathrm{n}=635$ ).

\begin{tabular}{|c|c|c|c|c|c|}
\hline & \multicolumn{2}{|c|}{ Média (DP) } & IC95\% & Sk & $\mathbf{K u}$ \\
\hline \multicolumn{6}{|l|}{ Adiposidade } \\
\hline IMC & \multicolumn{2}{|c|}{$21,14(4,18)$} & $20,81-21,46$ & 0,96 & 0,85 \\
\hline $\mathrm{CC}$ & \multicolumn{2}{|c|}{$71,32(10,16)$} & $70,53-72,11$ & 0,95 & 0,85 \\
\hline RCEst & \multicolumn{2}{|c|}{$0,44(0,06)$} & $0,44-0,45$ & 1,02 & 0,86 \\
\hline Atividade física & \multicolumn{2}{|c|}{$5,06(1,20)$} & $4,97-5,16$ & $-0,30^{a}$ & $-0,62^{\mathrm{a}}$ \\
\hline Imagem corporal & \multicolumn{2}{|c|}{$0,40(1,44)$} & $0,28-0,51$ & 0,42 & 0,47 \\
\hline \multirow[t]{3}{*}{ Qualidade de vida } & & & $3,66-3,75$ & $-0,57$ & 0,31 \\
\hline & \multicolumn{5}{|c|}{ Frequência de hábitos alimentares inadequados na última semana (\%) } \\
\hline & $\mathbf{0}$ & 1 & 2 & 3 & 4 \\
\hline Ultraprocessados & $105(16,5)$ & $62(9,8)$ & $203(32,0)$ & $154(24,3)$ & $111(17,5)$ \\
\hline Guloseimas & $84(13,2)$ & $97(15,3)$ & $180(28,3)$ & $185(29,1)$ & $89(14,0)$ \\
\hline
\end{tabular}

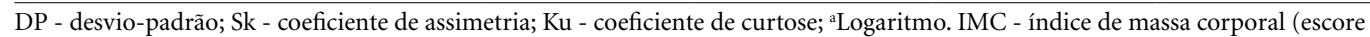
Z); CC - circunferência da cintura $(\mathrm{cm})$; RCEst - Razão cintura estatura $(\mathrm{cm})$. Todos os dias $=0$; De 4 a 5 vezes por semana $=1$; De 2 a 3 vezes por semana $=2$; Menos que 2 vezes por semana $=3$; Raramente ou nunca $=4$. 
Tabela 2. Coeficientes não padronizados, erro padrão e coeficientes padronizados do modelo com equações estruturais, utilizando a variável qualidade de vida como desfecho, entre adolescentes de 10 a 16 anos. Montes Claros, Minas Gerais, Brasil, 2016.

\begin{tabular}{|c|c|c|c|c|c|}
\hline & & & Coefic & ientes & \\
\hline & & itos & $\begin{array}{c}\text { Não } \\
\text { padronizados } \\
(\mathrm{EP})\end{array}$ & Padronizados & $\begin{array}{c}\text { p- } \\
\text { valor }\end{array}$ \\
\hline Qualidade de vida & & Atividade física & $0,10(0,02)$ & 0,21 & $* * x$ \\
\hline Qualidade de vida & & Imagem corporal & $0,02(0,02)$ & 0,04 & 0,330 \\
\hline Qualidade de vida & $\leftarrow$ & Hábitos alimentares inadequados & $-0,14(0,05)$ & $-0,17$ & 0,006 \\
\hline Qualidade de vida & $\leftarrow$ & Adiposidade & $-0,001(0,01)$ & $-0,004$ & 0,929 \\
\hline Atividade física & $\leftarrow$ & Hábitos alimentares inadequados & $0,05(0,09)$ & 0,03 & 0,579 \\
\hline Atividade física & $\leftarrow$ & Adiposidade & $0,01(0,01)$ & 0,02 & 0,617 \\
\hline Imagem corporal & & Atividade física & $0,05(0,05)$ & 0,04 & 0,346 \\
\hline Imagem corporal & & Hábitos alimentares inadequados & $0,01(0,11)$ & 0,01 & 0,896 \\
\hline Imagem corporal & & Adiposidade & $-0,03(0,02)$ & $-0,06$ & 0,124 \\
\hline $\begin{array}{l}\text { Hábitos alimentares } \\
\text { inadequados }\end{array}$ & & Adiposidade & $-0,02(0,01)$ & $-0,12$ & 0,041 \\
\hline Guloseimas & & Hábitos alimentares inadequados & 1,00 & 0,59 & \\
\hline Industrializados & $\leftarrow$ & Hábitos alimentares inadequados & $1,06(0,38)$ & 0,59 & 0,005 \\
\hline IMC & & Adiposidade & 1,00 & 0,90 & \\
\hline CC & & Adiposidade & $2,62(0,06)$ & 0,97 & $* * *$ \\
\hline RCEst & & Adiposidade & $0,02(0,00)$ & 0,93 & $* * *$ \\
\hline
\end{tabular}

EP - erro padrão; IMC - índice de massa corporal (escore Z); CC - circunferência da cintura (cm); RCEst - razão cintura estatura $(\mathrm{cm}) .{ }^{* *} \mathrm{p}<0,001$.

Fonte: Elaborado pelas autoras.

Tabela 3. Magnitude dos efeitos direto, indiretos e totais da variável independente adiposidade do modelo com equações estruturais, utilizando a variável qualidade de vida como dependente, entre adolescentes de 10 a 16 anos. Montes Claros, Minas Gerais, Brasil, 2016 ( $n=635$ ).

\begin{tabular}{rrlrrr}
\hline & & Efeitos & Direto & Indireto & Total \\
\hline Qualidade de vida & $\leftarrow$ & Adiposidade & 0,000 & 0,000 & 0,000 \\
& $\leftarrow$ & Atividade física & $0,209^{*}$ & 0,004 & $0,213^{*}$ \\
& $\leftarrow$ & Imagem corporal & 0,038 & 0,002 & 0,042 \\
& $\leftarrow$ & Hábitos alimentares inadequados & $-0,174^{*}$ & $0,020^{*}$ & $-0,150^{*}$ \\
\hline
\end{tabular}

${ }^{{ }^{\mathrm{p}}<0,05}$.

Fonte: Elaborado pelas autoras.

víduos menos ativos apresentaram 1,7 vezes mais risco de possuir domínio físico ruim do que seus pares mais ativos ${ }^{5}$. A situação inversa, também é verdadeira. Adolescentes inativos $(\mathrm{RC}=1,63$; $\mathrm{p}=0,05)$, obesos $(\mathrm{RC}=3,93 ; \mathrm{p}=0,05)$ apresentaram, mais chance de possuir percepção negativa quanto às relações sociais ${ }^{6}$. Já em outro estudo, adolescentes inativos ( $\mathrm{RC}=1,90$; IC95\% $=1,16$ $3,10)$, apresentavam-se ainda como prováveis dependentes de bebidas alcoólicas $(\mathrm{RC}=4,18$; IC95\%=1,04-16,84) e com mais chance de possuir percepção negativa do domínio psicológico 
bitos alimentares inadequados (pular o café da manhã $(\mathrm{OR}=1,56, \mathrm{P}=0,003)$, lanches irregulares $(\mathrm{OR}=1,43, \mathrm{P}<0,001)$ e o consumo frequente de macarrão instantâneo $(\mathrm{OR}=1,49, \mathrm{P}=0,007)$, dormir pouco $(\mathrm{OR}=1,15, \mathrm{P}=0,061)$ e inatividade física $(\mathrm{OR}=1,48, \mathrm{P}=0,022)^{25}$. Outra evidência aponta que a obesidade e EPC, consequentes a uma alimentação inadequada - marcada pelo consumo excessivo, especialmente de carboidratos e gorduras ${ }^{26}$-, podem resultar em doenças crônicas não transmissíveis (DCNT) e afetar significativamente a $\mathrm{QV}$ das pessoas ${ }^{27}$. Diante desses achados e dos resultados do presente estudo, torna-se coerente dizer que quanto mais frequentes forem os hábitos alimentares inadequados, pior será a QV dos adolescentes.

Ressalta-se que a associação entre QV e hábitos alimentares inadequados entre adolescentes não foi encontrada descrita na literatura. Dada tamanha importância desse tema, o investimento neste tipo de pesquisa permitirá conhecer amplamente os determinantes associados a essa díade, ampliando o impacto de ações de promoção, bem como sua eficiência para com práticas alimentares saudáveis, que influenciam positivamente na QV dos adolescentes.

Quanto à adiposidade, observou-se que esta exerceu efeito pequeno, negativo e significativo sobre hábitos alimentares inadequados. Tal achado contraria o estudo realizado com adolescentes sauditas para verificar associações entre medidas de obesidade e hábitos alimentares, dentre outros, que revelou que dentre os fatores que estiveram significativamente associados ao sobrepeso e à obesidade, houve destaque para o consumo bebidas açucaradas em 3-4 dias por semana $(\mathrm{OR}=1,27$; IC95\% $=1,05-1,53)$ ou $<3$ dias por semana $(\mathrm{OR}=1,32 \text {; } \mathrm{IC} 95 \%=1,08-1,62)^{28}$. Em outro estudo que visou identificar a prevalência excesso de peso e o consumo alimentar entre adolescentes de rede pública de ensino, houve similaridade entre os gêneros para com a prevalência de sobrepeso $(\mathrm{p}=0,826)$ e obesidade $(\mathrm{p}=0,276)$. Entretanto, $19,2 \%$ das meninas e $23,4 \%$ dos meninos exibiram consumo superior às recomendações de carboidratos ( $>65 \%$ do valor energético total) e $100 \%$ dos adolescentes consumiram ácidos graxos poliinsaturados em quantidades insuficientes, em contraste ao colesterol, cujo consumo estava acima das recomendações por $48 \%$ dos adolescentes ${ }^{29}$. No presente estudo, não foi possível identificar essa associação positiva entre adiposidade e hábitos alimentares inadequados, possivelmente em razão do viés de causalidade reversa, que pode ocorrer em estudos cujo deli- neamento é transversal. Deste modo, é possível supor que recomendações quanto à mudança de hábitos alimentares inadequados, incluindo os relacionados a gordura e carboidratos, podem ter resultado em maior concentração de adolescentes com excesso de peso e com menor ingestão desses alimentos, com vistas a melhorar sua condição de saúde. Sabe-se que a ingestão de alimentos e bebidas ultraprocessadas e prontos para consumo, como substitutos aos alimentos tradicionais e saudáveis, está associada ao aumento das prevalências de excesso de peso e de doenças crônicas já evidenciadas no público jovem ${ }^{30}$. Deste modo, a identificação de padrões alimentares ditos "não saudáveis" durante a infância e adolescência pode ser benéfico para o desenvolvimento de estratégias que melhorem os hábitos alimentares desses indivíduos e, consequentemente, diminuam a prevalência desses fatores de risco ao longo da vida ${ }^{31}$.

Embora no presente estudo tenha sido evidenciado que a AF e os hábitos alimentares inadequados tenham determinado mais fortemente a QV dos adolescentes investigados, a adiposidade parece impactar indiretamente na $\mathrm{QV}$, sendo esta mais afetada, na proporção em que o peso corporal aumenta ${ }^{11}$. Assim, seja diante do efeito direto da adiposidade ou mediado pelo estilo de vida dos adolescentes, a adiposidade exerce um importante papel na QV dos adolescentes investigados $^{6}$.

No modelo estrutural deste estudo, observou-se que a imagem corporal não contribuiu significativamente para a QV dos adolescentes. Pesquisadores apontam que adolescentes do sexo feminino (maioria no presente estudo), ao longo do tempo, modificam alguns valores comportamentais e adquirem uma visão crítica, nova e diferenciada, quanto à $\mathrm{QV}$, no que se refere a questões relacionadas à imagem corporal e auto estima $^{32}$, o que pode justificar a não associação da imagem corporal com a QV no presente estudo. Resultados similares foram encontrados no estudo que analisou, dentre outros, a associação entre imagem corporal e QV em distintos momentos da menarca de adolescentes (pré, pós-menarca e um ano após a menarca). No estudo citado, verificou-se que a insatisfação corporal também não se relacionou significativamente com a $\mathrm{QV}^{33}$.

Como aspectos positivos deste estudo, ressalta-se a utilização da modelagem com equações estruturais, por possibilitar examinar uma série de relações de dependência simultaneamente, indo além do que é feito usualmente nas análises estatísticas com técnicas clássicas ${ }^{20}$. Todavia, 
torna-se limitada as investigações avaliadas por meio de questionários, em detrimento de métodos diretos de mensuração, que podem subestimar ou superestimar as informações fornecidas pelos adolescentes investigados. Outra limitação deste estudo está relacionada ao método de máxima verossimilhança adotado para ajustar os modelos, o qual requer variáveis em escala de mensuração contínua. Entretanto, as variáveis utilizadas na composição do construto hábitos alimentares inadequados estão em escala com cinco categorias ordinais, ou seja, são variáveis que não comportam, em tese, uma análise paramétrica. Porém, ressalta-se que o método de máxima verossimilhança é robusto à violação do pressuposto da normalidade, desde que a assimetria e curtose das distribuições das variáveis não sejam muito elevadas ${ }^{20}$, e se o número de categorias das variáveis ordinais for, no mínimo, quatro $^{34}$. O viés da causalidade reversa inerente aos estudos transversais também limitou as análises deste estudo, já que possibilita estimar as associações entre as variáveis, mas não, estabelecer relações causais. Reforça-se que essa temática deva ser melhor explorada também, por meio de estudos longitudinais, com vistas a compreender melhor os fatores de risco envolvidos e traçar estratégias de prevenção.

Diante do exposto, este estudo evidenciou que a variável adiposidade apesar de não exercer efeito direto sobre a QV dos adolescentes, exerceu efeitos pequenos e positivos mediados pela $\mathrm{AF}$ e pequenos e negativos quando mediados por hábitos alimentares inadequados. Assim, verifica-se que os efeitos que a adiposidade exerce sobre a QV dos adolescentes, tendem a melhorar quando mediados pela prática de atividade física; bem como tendem a piorá-la quando mediados pelos hábitos alimentares inadequados. Já a atividade física exerceu efeitos pequenos e positivos sobre a QV dos adolescentes, tendendo a melhorá-la. Ressalta-se que o efeito de hábitos alimentares inadequados sobre a QV de adolescentes não foi encontrado descrito na literatura.

O presente estudo propiciou novas reflexões diante da complexidade que envolve a temática da adiposidade e demais fatores de risco, com suas repercussões na QV dos adolescentes. Assim, suscitam-se novas possibilidades para a investigação e compreensão deste fenômeno de modo direto e indireto.

\section{Colaboradores}

BIA Barbosa, ADG Fonseca e CSO Silva foram as idealizadoras do manuscrito. IA Barbosa, MF Silveira, L Pinho e MFSF Brito foram responsáveis pela metodologia e análise dos dados. Todos as autoras contribuíram para a redação, revisão, aprovação da versão final do manuscrito e são responsáveis pela totalidade do estudo, garantindo sua precisão e integridade.

\section{Referências}

1. Schmidt MI, Duncan BB, Silva GA, Menezes AM, Monteiro CA, Barreto SM, Chor D, Menezes PR. Chronic non-communicable diseases in Brazil: burden and current challenges. Lancet 2011; 377(9781):1949-1961.

2. Biro FM, Wien M. Childhood obesity and adult morbidities. Am J Clin Nutr 2010; 91(5):1499S-1505S.

3. Silva DAS, Petroski ELJ. The simultaneous presence of health risk behaviors in freshman college students in Brazil. J Community Health 2012; 37(3):591-598.

4. Silva DA, Peres KG, Boing AF, González-Chica DA, Peres MA. Clustering of risk behaviors for chronic noncommunicable diseases: a population-based study in southern Brazil. Prev Med 2013; 56(1):20-24.

5. Gordia AP, Quadros TMB, Campos W, Petroski EL. Domínio físico da qualidade de vida entre adolescentes: associação com atividade física e sexo. Rev Salud Publica 2009; 11(1):50-61.

6. Gordia AP, Quadros TMB, Silva RCR, Campos W. Domínio social da qualidade de vida de adolescentes e sua associação com variáveis comportamentais, biológicas e sóciodemográficas. Rev Educ Fis UEM 2015; 26(3):451-463. 
7. Gordia AP, Silva RCR, Quadros TMB, Campos W. Variáveis comportamentais e sociodemográficas estão associadas ao domínio psicológico da qualidade de vida de adolescentes. Rev Paul Pediatr 2010; 28(1):2935

8. Gouveia MJ, Frontini R, Canavarro MC, Moreira H. Imagem corporal e qualidade de vida na obesidade pediátrica. Psic Saude Doenças 2016; 17(1):52-59.

9. Luiz AMG, Gorayeb R, Liberatore Júnior RDR. Avaliação de depressão, problemas de comportamento e competência social em crianças obesas. Est Psicol 2010; 27:41-48.

10. Cataneo C, Carvalho AMP, Galindo EMC. Obesidade e aspectos psicológicos: maturidade emocional, autoconceito, locus de controle e ansiedade. Psicol Reflex Crit 2005; 18(1):39-46.

11. Casas AG, Guillamón AR, García-Cantó E, García PLR, Pérez-Soto JJ, Marcos LT, López PT. Estado nutricional y calidad de vida relacionada con la salud en escolares del sureste español. Nutr Hos 2015; 31(2):737-743

12. Kish L. Survey Sampling. New York: John Wiley \& Sons; 1965.

13. Brasil. Ministério da Saúde (MS). Orientações para coleta e análise de dados antropométricos em serviços de saúde: norma técnica do sistema de Vigilância Alimentar e Nutricional - SISVAN. Brasília: MS; 2011.

14. Ravens-Sieberer U, Gosch A, Abel T, Auquier P, Bellach BM, Bruil J, Mick Power WD, Rajmil L, the European KIDSCREEN Group. Quality of life in children and adolescents: a European public health perspective. Soz Praventivmed 2001; 46(5):294-302.

15. Craig CL, Marshall AL, Sjöström M, Bauman AE, Booth ML, Ainsworth BE, Pratt M, Ekelund U, Yngve A, Sallis JF, Oja P. International Physical Activity Questionnaire: 12-country reliability and validity. Med Sci Sports Exerc 2003; 35:1381-1395.

16. Brasil. Ministério da Saúde (MS). Teste: como está a sua alimentação? In: Guia alimentar - como ter um alimentação saudável [Internet]. [acessado 2020 jul 17]. Disponível em: https://bvsms.saude.gov.br/bvs/publicacoes/guia_alimentar_alimentacao_saudavel.pdf.

17. Stunkard AJ, Sorensen T, Schulsinger F. Use of the Danish adoption register for the study of obesity and thinness. Res Publ Assoc Res Nerv Ment Dis 1983; 60:115-120

18. Ainsworth BE, Haskell WL, Herrmann SD, Meckes N, Bassett DR Jr, Tudor-Locke C, Greer JL, Vezina J, Whitt-Glover MC, Leon AS. Compendium of physicactivities: a second update of codes and met values. Med Sci Sports Exercal 2011; 43:1575-1581.

19. World Health Organization (WHO). Global Database on Child Growth and Malnutrition: growth reference data for 5-19 years [Internet]. Genebra: WHO; 2007 [acessado 2020 jul 17]. Disponível em: http://www. who.int/growthref/en/.

20. Marôco J. Análise de equações estruturais: fundamentos teóricos, software e aplicações. Lisboa: Report Number; 2010.

21. Kline RB. Principles and practice of structural equation modeling. $3^{\mathrm{a}}$ ed. New York: Guilford Press; 2011.

22. Spengler $\mathrm{S}$, Woll A. The more physically active, the healthier? The relationship between physical activity and health-related quality of life in adolescents: the MoMo study. J Phys Act Health 2013; 10:708-715.
23. Casey MM, Harvey JT, Telford A, Eime RM, Mooney A, Payne WR1. Effectiveness of a school-community linked program on physical activity levels and health -related quality of life for adolescent girls. BMC Public Health 2014; 14:649.

24. Rother RL, Rempel C. Qualidade de vida de adolescentes que praticam atividade física: uma revisão sistemática. Rev Dest Acad 2017; 9(3):76-86.

25. Wang H, Sekine M, Chen X, Yamagami T, Kagamimori S. Lifestyle at 3 years of age and quality of life (QOL) in first-year junior high school students in Japan: results of the Toyama Birth Cohort Study. Qual Life Res 2008; 17(2):257-265.

26. Gulati S, Misra A. Sugar intake, obesity, and diabetes in India. Nutrients 2014; 6(12):5955-5974.

27. Sazlina SG, Zaiton A, Nor Afiah MZ, Hayati KS. Predictors of health related quality of life in older people with non-communicable diseases attending three primary care clinics in Malaysia. J Nutr Health Aging 2012; 16(5):498-502.

28. Al-Hazzaa HM, Abahussain NA, Al-Sobayel HI, Qahwaji DM, Musaiger AO. Lifestyle factors associated with overweight and obesity among Saudi adolescents. BMC Public Health 2012; 12:354.

29. Pinho L, Flávio EF, Santos SHS, Botelho ACC, Caldeira AP. Excesso de peso e consumo alimentar em adolescentes de escolas públicas no norte de Minas Gerais, Brasil. Cien Saude Colet 2014; 19(1):67-74

30. Sparrenberger K, Friedrich RR, Schiffner MD, Schuch I, Wagner MB. Ultra-processed food consumption in children from a Basic Health Unit. J Pediatr 2015; 91:535-542.

31. Ambrosini GL, Huang RC, Mori TA, Hands BP, O'Sullivan TA, Klerk NH, Beilin LJ, Oddy WH. Dietary patterns and markers for the metabolic syndrome in Australian adolescents. Nutr Metab Cardiovasc Dis 2010; 20:274-283.

32. Gordia AP, Quadros TMB, Campos W, Vilela Júnior GB. Qualidade de vida de adolescentes da rede particular de ensino: comparação entre gêneros. Rev Bras Qualid Vida 2009:1(2):16-24.

33. Santos MLB, Novaes JS, Mon teiro LAC, Fernandes HM. Insatisfação corporal e qualidade de vida durante a menarca e sua relação com a renda familiar e o índice de massa corporal: Um estudo longitudinal. Motri 2015; 11(2):75-84.

34. Oliveira BH, Nadanovsky P. Psychometric properties of the Brazilian version of the Oral Health Impact Profile-short form. Community Dent Oral Epidemiol 2005; 33:307-314.

Artigo apresentado em 16/04/2020

Aprovado em 08/09/2020

Versão final apresentada em 10/09/2020

Editores-chefes: Romeu Gomes, Antônio Augusto Moura da Silva 